\title{
BENCHMARKING DE QUALIDADE E SUSTENTABILIDADE DA PRODUÇÃO CIENTÍFICA EM ADMINISTRAÇÃO
}

\author{
Data de submissão: 10/10/2018 \\ Aceite: 01/11/2018 \\ Claudia Viviane Viegas ${ }^{1}$ \\ Angela Cristina Corrêa ${ }^{2}$ \\ Christianne Coelho de Souza Reinisch Coelho ${ }^{3}$ \\ Samara Rech ${ }^{4}$ \\ Vitor Francisco Schuch Júnior ${ }^{5}$ \\ Paulo Maurício Selig 6
}

\section{RESUMO}

O objetivo deste artigo é elaborar um referencial que sirva de apoio à qualificação da produção de artigos em Administração e a partir do qual possa ser proposto um benchmarking de qualidade e sustentabilidade da produção acadêmica nesta área. Pontualmente, visa contribuir para a qualificação de trabalhos a serem submetidos a periódicos nacionais Qualis A ou B da Coordenação de Aperfeiçoamento de Pessoal de Nível Superior (CAPES). Parte-se de análise bibliográfica sobre a situação da pesquisa em Administração segundo publicações em sete periódicos nacionais da área, ranqueados como $\mathrm{A}$ ou $\mathrm{B}$. Posteriormente é feita uma compilação dos critérios disponibilizados a autores pareceristas desses periódicos e de outros três internacionais, da área de Administração, para avaliar a produção a eles submetida. A revisão bibliográfica resultou na seleção de 51 artigos que apontam os principais problemas da pesquisa nesta área. Conclui-se que os critérios de avaliação dos periódicos coincidem com os mencionados em artigos selecionados, mas não permitem explorar aspectos da avaliação subjacentes às práticas blind e peer review. A melhoria contínua dos artigos é recomendada internacionalmente. O resultado é um quadro de referência para auxiliar na construção de um sistema de benchmarking de submissão em periódicos bem qualificados pela CAPES.

Palavras-chave: qualidade, produtividade, produção científica, administração, sustentabilidade.

\footnotetext{
1 Possui graduação em Geografia pela Universidade de Franca, UNIFRAN, graduação em Comunicação Social Habilitação Em Jornalismo pela Universidade do Vale do Rio dos Sinos, UNISINOS, mestrado em Administração de Empresas pela Universidade Federal do Rio Grande do Sul, UFRGS e doutorado em Engenharia e Gestão do Conhecimento pela Universidade Federal de Santa Catarina, UFSC. Florianópolis-SC. Brasil. E-mail: cldviegas@gmail.com 2 Possui graduação em Administração de Empresas pela Universidade Federal de Santa Maria, UFSM, mestrado em Engenharia de Produção pela UFSM e doutorado em Engenharia de Produção pela Universidade Federal de Santa Catarina, UFSC. Florianópolis-SC. Brasil. E-mail: angelaccorrea@gmail.com 3 Possui graduação em Engenharia Química pela Universidade Federal de Santa Catarina, UFSC, graduação em Museologia pela UFSC, mestrado em Engenharia de Produção pela UFSC e doutorado em Engenharia de Produção pela UFSC. Florianópolis -SC. Brasil. E-mail: christianne.ccsrc@gmail.com 4 Possui graduação em Ciências Econômicas pela Universidade Federal de Santa Catarina, UFSC e mestrado em Administração pela UFSC. Florianópolis-SC. Brasil. E-mail: samararech@hotmail.com

5 Possui graduação em Administração e Pedagogia pela Universidade Federal de Santa Maria, UFSM, mestrado em Administração pela Universidade Federal do Rio Grande do Sul, UFRGS e doutorado em Educação pela Universidade Estadual de Campinas, UNICAMP. Santa Maria - RS. Brasil. E-mail: vfschuch@gmail.com

6 Possui graduação em Engenharia Mecânica pela Universidade Federal do Rio Grande do Sul, UFRGS, mestrado em Engenharia de Produção pela Universidade Federal de Santa Catarina, UFSC e doutorado em Engenharia de Produção pela UFSC. Florianópolis-SC. Brasil. E-mail: pauloselig@gmail.com
} 


\section{INTRODUÇÃO}

A avaliação de periódicos é um assunto que tem gerado grandes debates e polêmicas, dado seu caráter abrangente e que varia de acordo com as percepções de cada avaliador. Além disso, a forma de cada avaliação é distinta em cada periódico. São diversos fatores que influenciam a avaliação de trabalhos. Este estudo aborda pontualmente a avaliação de periódicos na área de Administração. Amparado na ideia proposta por Kneipp et al. (2013) de que os periódicos científicos possuem importante papel na consolidação de determinada área do conhecimento, dado sua característica de divulgar e difundir resultados de pesquisas acadêmicas.

O objetivo deste artigo é elaborar um referencial que sirva de apoio à qualificação da produção de artigos em Administração e a partir do qual possa ser proposto um benchmarking de qualidade e sustentabilidade da produção acadêmica nesta área. Neste sentido, o objetivo está alinhado aos propósitos do projeto "Mapa Estratégico da Educação Superior (MEES) fundamentado em um sistema de gestão integrado: uma proposta metodológica para a operacionalização do PDI (Plano de Desenvolvimento Institucional)" (CORRÊA, et al, 2009).

O chavão "publicar ou perecer" é cada vez mais atual no meio acadêmico, tendo suas consequências expressas pelos constantes refinamentos de critérios de avaliação de programas de pós-graduação pela CAPES, os quais vêm reforçando a necessidade de estreitamento de laços entre pesquisadores de diferentes programas por meio de publicações em coautorias (SERRA et al., 2008). Seguindo essa linha de pensamento, Alcadipani (2011); Rossoni (2013) e Cabral e Lazzarini (2011) argumentam que a produção acadêmica brasileira se transformou em uma busca incessante por pontos.

Benchmarking é um processo contínuo de avaliação de produtos, serviços ou práticas gerenciais (Camp, 1997) buscando a identificação do que é melhor adotar para cumprir metas propostas. O processo de benchmarking, portanto, implica a identificação de referenciais sistematizáveis que possam ser acompanhados e melhorados ao longo do tempo. Kyrö (2003) observa que o benchmarking pode ser realizado em relação a um processo - como, por exemplo, o que envolve o contexto de produção, submissão e avaliação de artigos acadêmicos - e traz consigo a prática de aprendizagem.

Em trabalho no qual utilizou benchmarking comparativo, Sartori (2013) conclui que o Brasil está desenvolvido em relação a avaliações no âmbito da Comissão de Aperfeiçoamento de Pessoal de Nível Superior (CAPES).

Três fenômenos justificam a necessidade de investigar e propor soluções para um sistema de benchmarking voltado a referenciar a qualificação da publicação em Administração:

- o crescimento da quantidade de publicações em anais de eventos e em periódicos sujeitos aos critérios do sistema Qualis/CAPES (WOOD e CHEUKE, 2008), o que influencia a pontuação do ranking da pós-graduação no Brasil;

- a concentração de publicações qualificadas de autoria de pesquisadores de poucos programas de pós-graduação em Administração (OLIVEIRA e SAVERBRONN, 2007);

- o aumento do número de programas de pós-graduação em Administração no Brasil, de $168 \%$, entre 1998 e 2009 (ROSSONI e GUARIDO, 2009).

Além destes fenômenos, observa-se a ausência de critérios suficientemente detalhados para avaliadores (na submissão) e revisores (nos sistemas de pareceres).

Segundo Sola e Bonacim (2013), a partir da análise de periódicos brasileiros, constatouse que há concentração da produção científica na região Sudeste. 
Finalmente, há que se considerar a importância de melhor conhecer a situação brasileira no contexto de publicações em Administração, não apenas pelo mapeamento de meta-estudos em áreas específicas, como já ocorre, de modo a atualizá-los, mas principalmente pela necessidade de estruturação de um referencial uniforme para o sistema de publicações, o qual possibilite comparações adequadas da posição e dos desafios brasileiros quanto à publicação, comparativamente à situação presente no Exterior.

\section{MÉTODO}

O presente estudo caracteriza-se como uma pesquisa qualitativa de caráter exploratório, descritiva em suas formas de investigação e prescritiva na forma de apresentação de resultados. Sua contribuição está em buscar a sistematização do tecido de fatores que pode contribuir para a qualidade e a sustentabilidade da produção e da publicação de artigos nesta área.

O método prático de investigação da presente proposta constitui-se de uma revisão de normas para publicações emitidas por corpos editoriais de journals indexados pelo sistema Qualis/CAPES, sete nacionais e três internacionais, e de artigos sobre pesquisas em Administração veiculados nos periódicos nacionais selecionados.

Neste sentido, um levantamento bibliográfico foi realizado em duas instâncias: artigos publicados nos principais periódicos nacionais, Qualis A e B da grande área de Administração, Ciências Contábeis e Turismo, e junto aos sítios eletrônicos onde esses mesmos periódicos mantêm instruções a autores e/ou a pareceristas. Paralelamente, foram selecionados três journals internacionais, também da área de Administração, Ciências Contábeis e Turismo, todos Qualis A, para a análise dos critérios que eles disponibilizam para pareceristas e autores, visando a uma comparação entre essas diretrizes e as correspondentes dos journals nacionais da área.

A listagem a seguir apresenta os sete periódicos nacionais selecionados com os seus respectivos conceitos atribuídos pelo sistema Qualis/CAPES, no período de coleta de dados da pesquisa, ou seja, no ano de 2010, bem como informações complementares pertinentes.

1a) Revista de Administração Contemporânea (RAC), ISSN 1415-6555, Qualis B1 em Administração, Ciências Contábeis e Turismo (RAC, 2010), revisadas todas as edições disponíveis online, de 1997 a julho de 2010;

2a) Revista de Administração de Empresas da Fundação Getúlio Vargas (RAE/FGV), ISSN: 00347590, Qualis B1 nas áreas de Administração, Ciências Contábeis e Turismo, revisadas todas as edições disponíveis online, de 1961 a julho de 2010;

3a) Revista de Gestão da Universidade de São Paulo (RAUSP), ISSN 1516-7747, Qualis B3 em Administração, Ciências Contábeis e Turismo, revisadas todas as edições disponíveis online, de 1994 a julho de 2010;

4a) Revista de Administração Pública (RAP), ISSN 0034-712, Qualis A2 em Administração, Ciências Contábeis e Turismo, revisadas todas as edições publicadas no sistema Scielo, versão online desde 2006 a julho de 2010.

5a) Revista de Administração Mackenzie (RAM), ISSN 1678-6971, Qualis B 1 nas áreas de Administração, Ciências Contábeis e Turismo, revisadas todas as edições disponíveis online, desde 2000 a julho de 2010.

6a) Organizações e Sociedade (OeS), ISSN: 1413-585X, Qualis B2 em Administração, Ciências Contábeis e Turismo, revisadas todas as edições disponíveis online, no período de 2000 a julho de 2010;

7ạ) Organizações Rurais e Agroindustriais (OReA), ISSN 1517-3879, Qualis B2 em Administração, Ciências Contábeis e Turismo, revisadas todas as edições disponíveis online, desde 1996 até julho de 2010. 
A partir do levantamento nos periódicos nacionais selecionados, foi obtida uma compilação do conteúdo de 51 artigos sobre pesquisa em Administração, a qual foi classificada por similaridade de abordagem e conteúdo, e uma síntese de normas e recomendações a autores dos respectivos periódicos. Os journals internacionais foram explorados, para a finalidade desta pesquisa, unicamente com relação aos critérios de submissão para autores e revisão para editores.

\section{RESULTADOS}

Partindo do estado da arte sobre as publicações na área de Administração, o estudo permitiu apontar alguns resultados. A seção 3.1 apresenta as principais recomendações dos autores, em que são apontadas as fragilidades e iniciativas proativas com o intuito de promover a qualidade e sustentabilidade da qualidade do conhecimento produzido e disseminado.

\subsection{Síntese das recomendações e critérios para as publicações na área de administração}

\section{1a) Interdisciplinaridade nos meios, não nos fins}

Os benefícios da interdisciplinaridade na educação estão amparados na capacidade dos alunos de estabelecer diálogos entre os conteúdos (CEZARINO, 2013), porém os resultados são predominantemente descritivos e refletem bem mais a reprodução do conhecimento existente do que a análise na direção da criação de conhecimento novo (BULGACOV e VERDU, 2001; ROSSONI e GUARIDO, 2009; ALCADIPANI, 2013). Na graduação, este status é agravado pela falta de inter-relação entre as disciplinas do currículo, já que as instituições de ensino privilegiam o ensino em detrimento da pesquisa (NICOLINI, 2003; OLIVEIRA e SAVERBRONN, 2007).

Um fator positivo neste contexto é que há preocupação da comunidade acadêmica em analisar a produção científica nacional em diversas áreas da Administração (GRAEML e MACADAR, 2010), dada a massa considerável de meta-pesquisas.

\section{2a) Academicismo, descontinuidade e fragilidade teórica nas especialidades}

O que se verifica na pesquisa geral em Administração reflete-se em subáreas específicas: predomínio de autores internacionais e distanciamento da produção acadêmica relativamente à realidade empresarial. Contudo, há especificidades como baixa produção em algumas subáreas.

Em Aprendizagem Organizacional, a pesquisa está concentrada em poucas instituições. Segundo Takashi e Fischer (2009), 72\% de 43 artigos publicados nos principais periódicos nacionais de Administração são da UFBA, USP, Unisinos, UFSC, UFPR e UFRGS.

A participação da área de Contabilidade na pesquisa em Administração é baixa: 2,95\% em relação ao total de artigos publicados entre 1990 e 2003 em periódicos Qualis A CAPES, segundo análise de 2.037 artigos realizada por Cardoso, Mendonça, Riccio e Sakata (2005). Além disto, conforme estes autores, os artigos desta temática publicados em periódicos Qualis A têm baixa produção teórica e são pouco estruturados. Mendonça et al. (2009) corroboram a baixa produtividade da pesquisa brasileira na área de Contabilidade em relação ao Exterior.

Em resumo, observa-se que, quando registrado, o crescimento quantitativo da publicação nacional, nas especialidades da Administração, é descontinuado e/ou apresenta fragilidades em suas bases teóricas. 


\section{3a) Influência norte-americana desproporcional à qualidade do debate}

A influência norte-americana na pesquisa brasileira em Administração é constatada por diversos autores (BULGCOV e VERDU, 2001; VERGARA e PINTO, 2001; MACHADO-DA-SILVA et al., 2008; ALCADIPANI e CALDAS, 2012; ALCADIPANI e BERTERO, 2012; VALE et al., 2013; BERTERO et al., 2013). Segundo Serra et al. (2008), a maior parte das citações em pesquisas de Administração é estrangeira (52,4\%), segundo análise de 4.081 artigos publicados em anais do Encontro Nacional da Associação Nacional de Pós-graduação e Pesquisa em Administração (ENANPAD) e nas principais revistas científicas de Administração do Brasil revisados por Machado-da-Silva et al. (2008). Este fenômeno é verificado especialmente na literatura estrangeira de pesquisa sobre Aprendizagem Organizacional (TAKASHI e FISCHER, 2009). A questão da nacionalidade das citações não seria tão problemática na produção acadêmica se os artigos dela derivados fossem mais analíticos do que reprodutores de conhecimentos exógenos.

No Brasil, segundo Pinto e Lara (2008), como resultado da importação de teorias que foram desenvolvidas nos países centrais, é possível notar a defasagem de ideias que mostram uma compreensão tardia da realidade.

\section{4a) Graduação, o início da falta de cultura em pesquisa}

Em geral, estudantes de graduação são avessos à metodologia da pesquisa, conforme estudo realizado junto a 124 acadêmicos de instituições de ensino superior em Administração em Fortaleza (Ceará), realizado por Costa e Soares (2008). Embora não haja como correlacionar essas pesquisas, tampouco generalizar estes resultados, eles são claras demonstrações de obstáculos ao desenvolvimento de uma cultura de produção de artigos já na graduação.

Em contrapartida, estudantes de graduação consideram que os atributos de um docente bem qualificado incluem a publicação de artigos científicos, segundo entendimento de 214 entrevistados de 24 instituições de ensino em Administração de Curitiba (MACIEL, HOCAYEN-DASILVA e CASTRO, 2008).

\section{5a) Recorrência ao estudo de caso e fragilização da rastreabilidade}

Segundo Fleury (2003), estudos de caso correspondem a 32\% dos artigos publicados na RAP durante onze anos. Esta proporção é ainda maior quando se consideram as publicações dos anais do ENANPAD: 55,7\% dos 549 artigos deste evento, veiculados entre 2000 e 2005, nas áreas de Administração Pública e Gestão Social, usaram estudo de caso, conforme Hocayen-da-Silva et al. (2008).

\section{6a) Coautorias e redes: estratégia recente perante os critérios de avaliação da CAPES}

Serra et al. (2008) observam que há poucas parceiras entre brasileiros e estrangeiros para publicações em Administração.

Pode-se afirmar que a integração planejada dos pesquisadores para fins de publicação é incipiente. Estudo recente junto a 32 programas de pós-graduação em Administração no Brasil mostra que a rede de pesquisas entre eles é fraca, pouco densa, com relações esparsas; a localização geográfica e as linhas de pesquisa são importantes na definição da estrutura das redes; as linhas de pesquisa com maior grau de compartilhamento são: Organizações, Comportamento Organizacional e Estratégia (GUIMARÃES, GOMES, ODELIUS, ZANCAN e CORRACHI, 2009). Contudo, o número de 
coautorias nos programas de pós-graduação stricto sensu aumentou entre 2000 e 2006, principalmente devido a mudanças nos critérios de avaliação da CAPES, ou em função da formação de novos grupos e/ou do amadurecimento dos já existentes (MELLO, CRUBELLATTE e ROSSONI, 2009; 2010).

De fato, os pesquisadores estão trocando a autoria individual pela coletiva (GRAEML e MACADAR, 2010; Leal et al., 2013) porque o sistema de avaliação da CAPES motiva alto grau de desempenho quantitativo de produção intelectual, o que demanda a formação de grupos de cooperação para publicações (MACCARI et al., 2009; ROSSONI e GUARIDO, 2009; LEAL et al., 2013).

Mas, para evitar os fenômenos da endogenia e da excessiva concentração da pesquisa em programas com maior qualificação no sistema CAPES, são necessárias políticas institucionais que valorizem e fortaleçam a rede de programas de pós-graduação, incentivando pesquisas conjuntas, conforme recomendam Guimarães et al (2009). Constata-se que programas com maior produção intelectual tendem a interagir mais entre si, e programas periféricos tendem a se relacionar com os centrais.

\section{7a) Processo de avaliação de artigos: falta clareza}

O processo de avaliação de artigos em periódicos de elevada classificação no sistema Qualis/CAPES gera muitas discussões entre pesquisadores. Em geral, os editores adotam os métodos blind e doublereview, que correspondem, respectivamente, à avaliação cega, sem identificação dos autores para os pareceristas e vice-versa, e à revisão por pares - mais de um avaliador, os quais não são identificados entre si para efeito de cada trabalho de avaliação.

O sistema de avaliação cega é criticado sobretudo sob o argumento de que o anonimato confere um poder outorgado sem mérito ao parecerista (THIRY-CHERQUES, 2005).

As elevadas taxas de rejeição também desencorajam autores. Conforme Serra et al. (2008), este não é um fenômeno isolado, pois, por exemplo, o Science Quarterly, na área de Administração, tem uma taxa de rejeição de artigos de pelo menos 90\%. E o Academy of Information and Management Journal apresenta média de aceitação de 25\% (AIMJ, 2010).

Uma das questões mais relevantes neste contexto é como aumentar as taxas de publicação sem afrouxar critérios de qualidade na submissão. Nem todos os periódicos bem classificados no Qualis/CAPES, na área de Administração, possuem recomendações claras para os autores, o que facilitaria o enquadramento dos artigos às exigências do periódico.

\section{8a) Critérios e recomendações de autores nacionais para a qualidade da pesquisa}

Os principais pontos em comum quanto aos critérios de aceitação de um artigo, conforme os autores revisados em 51 pesquisas publicadas nas principais revistas nacionais de Administração com elevada classificação pelo sistema Qualis/CAPES, são o rigor teórico e metodológico e a contribuição científica para a teoria que o artigo de fato representa (POZZEBON e FREITAS, 1998; BERTERO et al, 2003; PFEFFER e FONG, 2003; SANTOS E ICHIKAWA, 2003; HOPPEN e MEIRELLES, 2005; VIEIRA, 2007; SERRA et al, 2008; BERTERO et al, 2013). Uma avaliação propositiva e detalhada é sugerida por Pinho (2005). Bertero et al (2013), propõem oito elementos que possibilitem reflexão, além de um possível norteamento na pesquisa: foco no Brasil e na realidade local; aproximação da teoria e prática; estabelecer planos de pesquisa buscando aprofundamento em temas; fomentar o rigor científico; focar no impacto da produção; dar privilégio a periódicos internacionais de nível alto; reformar os programas de pós-graduação; reconhecer pesquisadores e pesquisas exemplares. 


\section{9a) Critérios e recomendações de editores de periódicos nacionais e internacionais}

Os critérios comuns exigidos por editores e apresentados nos sites dos periódicos nacionais são: ineditismo; atendimento a políticas editoriais do veículo; validade interna e externa da pesquisa; robustez teórica e metodológica; e apresentação de contribuição relevante para o conhecimento (RAC, 2010; RAE/ FGV, 2010; RAM, 2010; RAP, 2010; RAUSP, 2010; O\&S, 2010; OR\&A, 2010). Observam-se correspondências entre critérios e recomendações presentes na literatura em Administração e em sites de periódicos.

No que diz respeito aos periódicos internacionais considerados, dois deles não apresentam, em seus sites, informações claras sobre o processo de avaliação de artigos: BSE e CJAS. Ambos são da base Wiley, que adota sistema triple blind review para suas publicações e mantém instruções técnicas (tutorial) para formatação e submissão de artigos. Já o AIMJ, da base Allied Academies, traz instruções para autores e revisores. Para os primeiros, a principal recomendação é a completude da revisão bibliográfica e a consistência metodológica, assim como a adequação dos constructos às conclusões. Para revisores, a principal recomendação é serem propositivos, indicando especificamente o que deve ser feito para possibilitar a publicação do artigo.

Comparando-se brevemente periódicos nacionais e internacionais quanto ao sistema de avaliação e recomendações a revisores, nota-se que a política editorial de suporte ao autor, no sentido de encorajar a melhoria contínua, buscando efetivar a publicação, aparece como um diferencial importante no AIMJ, não estando necessariamente presente nas políticas editoriais dos periódicos nacionais.

\subsection{Benchmarking de apoio à qualidade e sustentabilidade da publicação}

Na presente proposta, a sistematização de elementos para um benchmarking de apoio à qualificação e sustentabilidade da publicação em Administração insere-se no escopo do projeto MEES (CAPES, 2009) e está ancorada em quatro questionamentos: dois para pesquisadores em geral, um para autores e outro para avaliadores. Para pesquisadores, as perguntas-chave consideradas são "O que superar?" e "Como criar sinergia para melhorar a pesquisa?". Para autores e avaliadores, a pergunta é a mesma: "O que seguir?". Estes questionamentos derivam do resultado da revisão bibliográfica e das lacunas encontradas ao logo da mesma quanto à busca de respostas para tornar mais objetivo e efetivo o processo que vai da produção à submissão e avaliação de artigos.

Os quadros 1, 2 e 3 apresentam para os autores, avaliadores e pesquisadores em geral, respectivamente, os principais problemas recorrentes identificados nas pesquisas da área de administração, a sinergia para melhorar os resultados e uma abordagem prescritiva do que fazer para alcançar a excelência nas publicações.

Quadro 1: Problemas recorrentes

\begin{tabular}{|l|l|}
\hline \multicolumn{1}{|c|}{ O que superar } & \multicolumn{1}{c|}{ Referências } \\
\hline $\begin{array}{l}\text { Falta de interdisciplinaridade dos resultados da pesquisa em Ad- } \\
\text { ministração }\end{array}$ & Nicolini (2003), Bertero et al. (2013) \\
\hline Distância da pesquisa em relação à realidade das empresas & Vieira (2007); Bertero et al. (2013) \\
\hline Baixa valorização da produção nacional & Bignetti e Paiva (2002); Alcadipani (2011) \\
\hline Baixa valorização dos aspectos teóricos na pesquisa & (Vieira, 2007) \\
\hline Falta de cultura de pesquisa na graduação & Nicolini (2003); Oliveira e Saverbronn (2007) \\
\hline Falta de integração professor-aluno para publicar & Fleury (2003) \\
\hline Excessiva recorrência ao estudo de caso & Roesch (2003) \\
\hline Centralização das redes de pesquisa & Braga et al. (2008) \\
\hline $\begin{array}{l}\text { Endogenia - tendência a concentrar a publicação em periódicos } \\
\text { de instituições de ensino locais }\end{array}$ & Wood e Cheuke (2008) \\
\hline
\end{tabular}


Quadro 2: Sinergia para melhorar a pesquisa

\begin{tabular}{|l|l|}
\hline \multicolumn{1}{|c|}{ O que analisar } & \multicolumn{1}{c|}{ Referências } \\
\hline Estrutura da comunidade acadêmica para cooperar em publicação & Braga et al. (2008) \\
\hline Produção do conhecimento em rede: alcance e profundidade & $\begin{array}{l}\text { Bulgacov e Verdu (2001); Guimarães } \\
\text { et al. (2009) }\end{array}$ \\
\hline Potencial de internacionalização da publicação & Serra et al. (2008); Cabral et al (2013) \\
\hline Causas de rejeição de artigos em periódicos internacionais & Serra et al. (2008) \\
\hline $\begin{array}{l}\text { Impactos da experiência do avaliador sobre sua capacidade de } \\
\text { publicação }\end{array}$ & Serra et al. (2008) \\
\hline
\end{tabular}

Quadro 3: O que seguir? (Autores e avaliadores)

\begin{tabular}{|c|c|c|}
\hline \multicolumn{3}{|c|}{ Autores } \\
\hline O que buscar & Periódicos que exigem & Referência \\
\hline $\begin{array}{l}\text { Perspectiva teórica, robustez, indicação de avanços, } \\
\text { profundidade, epistemologia, bibliografia de periódicos } \\
\text { qualificados, nacionais e internacionais }\end{array}$ & $\begin{array}{l}\text { Nacionais: } 1 / 7 \text { periódicos } \\
\text { Internacionais: AIMJ }\end{array}$ & $\begin{array}{l}\text { Berteroet al. (2003); Hoppen e } \\
\text { Meirelles (2005); Vieira (2007); } \\
\text { Serra et al. (2008) }\end{array}$ \\
\hline $\begin{array}{l}\text { Método: rigor na condução de etapas da pesquisa, } \\
\text { análise de variáveis, interpretação de dados }\end{array}$ & $\begin{array}{l}\text { Nacionais: } 3 / 7 \text { periódicos } \\
\text { Internacionais: AIMJ }\end{array}$ & $\begin{array}{l}\text { Pozzebon e Freitas (1998); } \\
\text { Berteroet al. (2003); Hoppen e } \\
\text { Meirelles (2005); Vieira (2007) }\end{array}$ \\
\hline $\begin{array}{l}\text { Validade interna: constructos, método coerente com } \\
\text { técnicas de pesquisa empregadas }\end{array}$ & Nacionais: $4 / 7$ periódicos & Hoppen e Meirelles (2005) \\
\hline Validade externa: potencial de generalização & Nacionais: $6 / 7$ periódicos & Hoppen e Meirelles (2005) \\
\hline Locus e contexto: longitudinal ou cross seccional & & Pozzebon e Freitas (1998) \\
\hline Objetivos e redação claros & Nacionais: $4 / 7$ periódicos & Serra et al. (2008) \\
\hline $\begin{array}{l}\text { Algo a propor: originalidade, valor, contribuição, } \\
\text { aplicação prática, desafio ao pensamento corrente, } \\
\text { direcionamento à mudança }\end{array}$ & $\begin{array}{l}\text { Nacionais: } 3 / 7 \text { periódicos } \\
\text { Internacionais: AIMJ }\end{array}$ & $\begin{array}{l}\text { Pfeffer e Fong (2003); Santos } \\
\text { e Ichikawa (2003); Hoppen e } \\
\text { Meirelles (2005); Vieira (2007); } \\
\text { Serra et al. (2008) }\end{array}$ \\
\hline $\begin{array}{l}\text { Organização: desenho bem definido, delimitações, } \\
\text { unidades de análise, hipóteses }\end{array}$ & Nacionais: $1 / 7$ periódicos & Hoppen e Meirelles (2005) \\
\hline $\begin{array}{l}\text { Contribuição proporcional em caso de mais de um } \\
\text { autor; limite de submissão por autor }\end{array}$ & Nacionais: $4 / 7$ periódicos & Berteroet al. (2003) \\
\hline $\begin{array}{l}\text { Estudos de caso: ter perfil pedagógico, possível } \\
\text { estrutura hipertextual (diversas possibilidades de } \\
\text { aproveitamento), profundidade }\end{array}$ & $\begin{array}{l}\text { Nacionais: } 1 / 7 \text { periódicos } \\
\text { Internacionais: AIMJ }\end{array}$ & $\begin{array}{l}\text { Pozzobon e Freitas (1998); Lima } \\
\text { (2003); Ikeda et al. (2005) }\end{array}$ \\
\hline $\begin{array}{l}\text { Direcionamento da pesquisa para questões nacionais, } \\
\text { atualidade, oportunidade }\end{array}$ & Nacionais: $2 / 7$ & $\begin{array}{l}\text { Campos (1984); Vergara e Pinto } \\
(2001)\end{array}$ \\
\hline \multicolumn{3}{|c|}{ Revisores } \\
\hline O que buscar & Periódicos que exigem & Referência \\
\hline $\begin{array}{l}\text { Avaliação orientadora, propositiva, indicando o que } \\
\text { fazer para o artigo ser aceito }\end{array}$ & Internacionais: AIMJ & Gondim (2004); Pinho (2005) \\
\hline
\end{tabular}

A partir da pesquisa bibliográfica realizada e da análise comparativa dos conteúdos encontrados na literatura nacional e internacional considerada, é possível indicar os elementos dos Quadros 1 a 3 como relevantes para integrar um sistema de benchmarking de qualificação da publicação em Administração. 


\section{CONSIDERAÇÕES FINAIS}

Os achados da pesquisa permitem atingir o objetivo geral deste estudo. $O$ estado-da-arte apresentado por meio de uma síntese das recomendações e critérios para as publicações e a sistemática de avaliação reflexiva proposta nos Quadros 1 a 3 se constituem em relevantes contribuições para um benchmarking de apoio à qualidade e sustentabilidade das publicações na área de administração. E pode-se dizer que permitem algumas generalizações para as demais áreas do conhecimento. Verifica-se que os critérios comuns exigidos por editores são: ineditismo; atendimento a políticas editoriais do veículo; validade interna e externa da pesquisa; robustez teórica e metodológica; e apresentação de contribuição relevante para o conhecimento. Para os autores, os elementos mais importantes são: rigor teórico e metodológico; contribuição de valor; delineamento de objetivos compatíveis com a pesquisa; confiabilidade dos constructos; e impacto da pesquisa sobre a realidade. São apresentadas também recomendações de editores e autores para a qualificação da pesquisa em Administração e constatações sobre a situação atual da produção acadêmica na área segundo sete aspectos: perfil geral da pesquisa em Administração; influência norte-americana; formação do administrador e papel do docente na área; perfil da produção de artigos em áreas específicas da Administração; estudo de caso; integração entre pesquisadores, formação de redes de co-autorias e critérios CAPES de avaliação; pareceristas e processo de avaliação de artigos. Esses referenciais são correlacionados em um desenho de pesquisa focado na obtenção de um benchmarking nacional de produção e disseminação de pesquisa acadêmica na pós-graduação em Administração, o qual deve ser co-referenciado em padrões internacionais. Conclui-se que os critérios de avaliação dos periódicos coincidem com os mencionados em artigos selecionados, mas não permitem explorar aspectos da avaliação subjacentes às práticas blind e peer review. Para consolidar o benchmarking da qualidade e sustentabilidade da produção científica em Administrativa, faz-se as seguintes recomendações: ampliar a base de pesquisa em periódicos internacionais a respeito de critérios de avaliação, testá-los, refiná-los e atribuir uma escala de valoração aos elementos resultantes; realizar atividades de integração entre editores, pareceristas e avaliadores da CAPES, para discutir a possibilidade de um referencial harmônico de avaliação de artigos. Almeja-se que os resultados deste estudo e as recomendações sugeridas para futuros trabalhos possam contribuir para a melhoria da qualidade geral do sistema de publicações nacionais em Administração.

\section{REFERÊNCIAS}

AIMSJ. Academy of Information and Management Sciences Journal. Disponível em: <http:// www.alliedacademies.org/public/journals/JournalDetails.aspx?jid=10>, 2010. Último acesso: 10/07/2016.

ALCADIPANI, R. Resistir ao produtivismo: uma ode à perturbação acadêmica. Cadernos EBAPE. BR, Vol.9, N.4,p.1174-1178, 2011.

ALCADIPANI, R. O Estrangulamento da Produção do Conhecimento em Administração no Brasil: Produtivismo, Paroquialismo e Estrangeirismo. In: BERTERO, C.O., ALCADIPANI, R.; CABRAL, S.; FARIA, A.; ROSSONI, L. Os desafios da produção de conhecimento em Administração no Brasil. Cadernos Ebape/FGV, p.11, N.1, p.181-196, 2013.

ALCADIPANI, R; BERTERO, C.O. Guerra fria e ensino do management no Brasil: o caso da FGVEAESP. Revista de Administração de Empresas, Vol.52, n.3, p.284-299, 2012. 
BERTERO, C.O.; VASCONCELOS, F.C.; BINDER, M.P. Estratégia empresarial: a produção científica brasileira entre 1991 e 2002. Revista de Administração de Empresas/FGV, Vol.43, N.4, p.48-62, 2003.

BERTERO, C.O.; VASCONCELOS, F.C.; BINDER, M.P.; WOOD Jr, T. Produção Científica Brasileira em Administração na década de 2000. Revista de Administração de Empresas/FGV, Vol.53, N.1, p.12-20, 2013.

BIGNETTI, L.P.; PAIVA, E.L. Ora (direis) Ouvir Estrelas!: Estudo das citações de autores de estratégias na produção acadêmica brasileira. Curitiba: Revista de Administração Contemporânea, Vol.6, N 1, jan-abr: 105-125, 2002.

BRAGA, M.J.C.; GOMES, L.F.A.M.; RUEDIGER, M.A. Mundos pequenos, produção acadêmica e grafos de colaboração: um estudo de caso dos Enanpads. Rio de Janeiro: Revista de Administração Pública, Vol.42, N 1, jan-fev: 133-154, 2008.

BULGACOV, S.; VERDU, F.C. Redes de pesquisadores da área de administração: um estudo exploratório. Curitiba: Revista de Administração Contemporânea, Ed. Especial, p.163-182, 2001.

BSE. Business Strategy and the Environment. Disponível em: <http://onlinelibrary.wiley.com/ journal/10.1002/\%28ISSN\%291099-0836>. Último acesso: 04.Ago. 2015.

CABRAL; LAZZARINI, S.G. Internacionalizar é preciso, produzir por produzir não é preciso. Organizações e Sociedade, Vol.18,N.58, p.541-542, 2011.

CAMP, R.C. Benchmarking: the search for industry best practices that lead to superior performance.(1997). Disponível em: <http://www.portalbmk.org.br/publico/bench_ind.php>. Último acesso: 10.Set.2015.

CAMPOS, A.M. Pesquisa: relevância social, cooperação e abertura à aprendizagem. Rio de Janeiro: Revista de Administração de Empresas/FGV. Vol.24, N.4: 141-145, out-dez, 1984.

CJAS. Canadian Journal of Administrative Sciences. Disponível em: <http://onlinelibrary.wiley. com/journal/10.1002/\%28ISSN\%291936-4490>. Último acesso: 05.Ago.2016.

CARDOSO, R.L.; MENDONÇA NETO,; RICCIO, E.L.;SAKATA, M.C.G. Pesquisa científica em contabilidade entre 1990 e 2003. Revista de Administração de Empresas/FGV, Vol.45, N.2, p.34-45, 2005.

CEZARINO, L.O. Mensuração da interdisciplinaridade nos cursos de graduação em Administração. Tese. Universidade de São Paulo, São Paulo, 2013. 279p.

CAPES. Coordenação de Aperfeiçoamento de Pessoal de Nível Superior.Web Qualis, 2010. Disponível em: Contido em: <http://qualis.capes.gov.br/webqualis/ConsultaPeriodicos.faces>. Último acesso: 10.maio.2011.

CAPES. Coordenação de Aperfeiçoamento de Pessoal de Nível Superior. Resultado Edital № 09/C II 2008.(2009). Disponível em: <http://www.capes.gov.br/images/stories/download/editais/ Resultado_Edital09_ProAdministracao.pdf>. Último acesso: 10.maio.2011.

CORRÊA, A.C. Mapa Estratégico da Educação superior (MEES) fundamentado em um sistema de gestão integrada: uma proposta para a operacionalização do PDI. Edital PROADM/CAPES, 2009. Disponível em: <www.sigmees.com.br>. Último acesso: 30.maio.2010.

Costa, F.J., e Soares, A.A.C. Uma análise da formação científica em cursos de graduação em Administração: a perspectiva dos alunos. Revista de Gestão da USP, Vol.15, N.1, p.47-60, 2008. 
FLEURY, S. (Org.). Análise do perfil dos artigos publicados na Revista de Administração Pública RAP - no período 1992-2002. 2003. Disponível em: <http://www.ebape.fgv.br/academico/pdf/ RelRAP.pdf>. Último acesso: 10.maio.2010.

GONDIM, S. A face oculta do parecerista: discussões éticas sobre o processo de avaliação de mérito de trabalhos científicos. Organizações e Sociedade, Vol. 11, N. 31, set-dez: 195-199, 2004.

GRAEML; A.R.; MACADAR, M.A. Análise de citações utilizadas em ADI: 10 anos de anais digitais do Enanpad (1997-2006). Revista de Administração Contemporânea,V.14,n.1, p.122-148, 2010.

GUIMARÃES, T.A.; GOMES, A.O.; ODELIUS, C.C.; ZANCAN, C.; CORRACHI, A.A. A rede de programas de pós-graduação em Administração no Brasil: análise de relações acadêmicas e atributos de programas. Revista de Administração Contemporânea, Vol.13, N.4,p.564-582, 2009.

HOCAYEN-DA-SILVA; ROSSONI, L; FERREIRA JUNIOR, I. Administração pública e gestão social: a produção científica brasileira entre 2000 e 2005. Revista de Administração Pública, Vol.42, N.4, p.655-680, 2008.

HOPPEN, N.; MEIRELLES, F.S. Sistemas de Informação: um panorama da pesquisa científica entre 1990 e 2003. Revista de Administração de Empresas/FGV, Vol.45, N.1, p.24-35, 2005.

KNEIPP, J.M.; ROSA, L.A.B.; BICHETI, R.S., POZZER, R.H.P; SCHUCH JUNIOR, V.F. Análise bibliométrica da produção científica da Revista de Administração da UFSM: em busca de novas perspectivas e desafios. Revista de Administração da UFSM, Santa Maria, Vol.6, N.2, p.443-458, 2013.

KYRÖ, P. Revising the concept sand foruns of benchmarking. Benchmarking; an International Journal, Vol.10, N.3, p.210-225, 2003.

LEAL, R.P.C.; ALMEIDA, V.S.; BORTOLON, P.M. Produção científica brasileira em finanças no período 2000-2010. Revista de Administração de Empresas, Vol.3, N.1, p.46-55, 2013.

MACCARI, E.H., ALMEIDA, M.I.R., NISHIMURA, A.T.; RODRIGUES, L.C. A gestão dos programas de pós-graduação em Administração com base no sistema de avaliação da Capes. Revista de Gestão da USP, Vol.16, N.4, p.1-16, 2009.

MACHADO-DA-SILVA, C.L.; GUARIDO FILHO; E.R.; ROSSONI, L.; GRAEFF, J.F. Periódicos brasileiros de Administração: análise bibliométrica de impacto no triênio 2005-2007. Revista de Administração Contemporânea, Curitiba Vol.2, N.3, 351-373, set-dez. 2008.

MACIEL, C.O.; HOCAYEN-DA-SILVA, J.A.; CASTRO, M. O ideário de Escola na ótica dos docentes: pura subjetividade ou padrões estruturados de cognição nos cursos de Administração? Revista de Administração Contemporânea, Vol.12, N.3, p.659-688, 2008.

MELLO, C.M., CRUBELLATE, J.M.; ROSSONI, L. Redes de coautorias entre docentes de programas brasileiros de pós-graduação (stricto-sensu) em Administração: aspectos estruturais e dinâmica de relacionamento. Revista de Administração Mackenzie, Vol.10, N.5, p.130-153, 2009.

MELLO, C.M., CRUBELLATE; ROSSONI, L. Dinâmica de relacionamento e prováveis respostas estratégicas de programas brasileiros de pós-graduação em Administração à avaliação da Capes: proposições institucionais a partir da análise de redes de co-autorias. Revista de Administração Contemporânea, Vol.14, N.3, p.434-457, 2010. 
MENDONÇA, O.R.; NETO, R.E.L.; SAKATA, M.C.G. Dez anos de pesquisa contábil no Brasil: análise dos trabalhos apresentados nos Enanpads de 1996 a 2005. Revista de Administração de Empresas/FGV, Vol.49, N.1, p.62-72, 2009.

NICOLINI, A. Qual será o futuro das fábricas de administradores? Revista de Administração de Empresas/FGV, Vol.43, N.2, p.44-54, 2003.

OLIVEIRA, F.B.; SAVERBRONN, F.F. Trajetórias, desafios e tendências no ensino superior de Administração e Administração Pública no Brasil: uma breve contribuição. Revista de Administração Pública, Ed. Especial, p.149-170, 2007.

O\&S. Organizações e Sociedade. Políticas editoriais. 2010. Disponível em: <http://www. revistaoes.ufba.br/policies.php\#peer>. Último acesso: 09.maio.2011.

OR\&A. Organizações Rurais e Agroindustriais. Normas e orientações para publicação. 2010. Disponível em: <http://www.dae.ufla.br/revista/>. Último acesso: 09.maio.2010.

PFEFFER, J.; FONG, C.T. O fim das escolas de negócio? Revista de Administração de Empresas/ FGV, Vol.43, N.2, p.11-28, 2003.

PINHO, J.A.G. Brevíssimo Manual do Editor: considerações sobre submissão e avaliação de artigos. O papel dos pareceristas e do editor de revistas científicas. Organizações \& Sociedade, Vol.12, N.34, jul-set: 156-173, 2005.

PINTO, M.R.; LARA, J.E. O que se publica sobre comportamento do consumidor no Brasil, afinal? Revista de Administração da UFSM, Santa Maria, Vol., N.1, p.85-100, 2008.

POZZEBON, M.; FREITAS, H.M.R. Pela aplicabilidade - com maior rigor científico - dos estudos de caso em sistemas de informação. Curitiba: Revista de Administração Contemporânea, Vol.2, N.2, p.143-170, 1998.

RAC. Revista de Administração Contemporânea. Submissão de trabalhos. 2010. Disponível em: <http://www.anpad.org.br/rac/rac-page-normas-frame.html>. Último acesso: 20.out.2011.

RAE/FGV Revista de Administração de Empresas da Fundação Getúlio Vargas. Orientações para autores.2010. Disponível em: <http://www16.fgv.br/rae/rae/index. cfm?FuseAction=OrientacoesAutores $>$. Último acesso: 15.out.2011.

RAM. Revista de Administração Mackenzie. Política editorial. 2010. Disponível em: <http:// www3.mackenzie.br/editora/index.php/RAM/about/editorialPolicies\#focusAndScope>. Último acesso: 12.nov.2011.

RAP. Revista de Administração Pública. Normas para publicação. 2010. Disponível em: <http:// www.ebape.fgv.br/academico/asp/dsp_rap_normas.asp>. Último acesso: 10.nov.2011.

RAUSP. Revista de Gestão da Universidade de São Paulo. Área de acesso - Reservado a autores e avaliadores.2010. Disponível em: <http://www.rausp.com.br/publicacao/index.asp>. Último acesso: 15.dez.2011.

ROESCH, S.M.A. Quem responde pelo desempenho limitado da produção científica em Administração no Brasil? Organizações \& Sociedade, Vol. 10, N. 28, set-dez: 165-167, 2003. 
ROSSONI, L; HOCAYEN-DA-SILVA, J.A. Aspectos estruturais da cooperação entre pesquisadores no campo da Administração Pública e Gestão Social: análise das redes entre instituições no Brasil. Revista de Administração Pública, Vol.42, N.6, p.1041-1067, 2008.

ROSSONI, L. Os desafios da Academia Brasileira em Administração enquanto Empreendimento Social. In: Bertero, C.O., Alcadipani, R., Cabral, S., Faria, A., Rossoni, L. Os desafios da produção de conhecimento em Administração no Brasil. Cadernos Ebape/FGV, Vol.11, N.1, p.181-196, 2013.

ROSSONI, L.; GUARIDO FILHO, E.R. Cooperação entre programas de pós-graduação e, Administração no Brasil: evidências estruturais em quatro áreas temáticas. Revista de Administração Contemporânea, Vol.3, N.3, p.366-390, 2009.

SANTOS, L.W.; ICHIKAWA, E.Y. Ciência, tecnologia e sociedade. Visões sobre transformações da pesquisa agrícola no Brasil. Organizações Rurais e Agroindustriais - Revista de Administração da UFLA, Vol. 5, N.2, p.66-79, 2003.

SARTORI, T. Avaliação comparativa na pós-graduação stricto sensu Brasil e Portugal: um estudo de caso na engenharia civil. Dissertação. Programa de Pós-graduação em Engenharia de Produção - Universidade Federal de Santa Maria. 2013. 127p.

SERRA, F.A.R.; FIATES, G.G.; FERREIRA, M.P. Publicar é difícil ou faltam competências? O Desafio de pesquisar e publicar em revistas científicas na visão de editores e revisores internacionais. Revista de Administração Mackenzie, São Paulo, Vol.9, N.4, Ed. Especial: 32-55, 2008.

SOLA, G.L.; BONACIM, C.A.G. Avaliação bibliométrica de periódicos brasileiros: contrastando a metodologia Qualis-Capes com o modelo de Krzyzanowski e Ferreira (1998). Revista de Administração da UFSM, Santa Maria, Vol.6, N.1, p.55-70, 2013.

TAKASHI, A.R.W.; FISCHER, A.L. Debates passados, presentes e futuros da Aprendizagem Organizacional - um estudo comparativo entre a produção acadêmica nacional e internacional. Revista de Administração Mackenzie, Vol. 10, N.5, p.53-76, 2009.

THIRY-CHERQUES, H.R. Comentando: "A face oculta do parecerista: discussões éticas sobre o processo de avaliação de mérito de trabalhos científicos". Organizações e Sociedade, Vol.12,N.3, p.181-182, 2005.

VALE, M.P.E.M.; BERTERO, C.O.; SILVEIRA, R.A. Caminhos diferentes da americanização na educação em Administração no Brasil: a EAESP/FGV e a FEA/USP. Administração: Ensino e Pesquisa, Vol.14, N.4, p.837-872, 2013.

VERGARA, S.C.; PINTO, M.C.S. Referências teóricas em análise organizacional: um estudo das nacionalidades dos autores referenciados na literatura brasileira. Revista de Administração Contemporânea, Edição Especial, p.102-121, 2001.

VIEIRA, V.A. Proposta de critérios para avaliação de dissertações de mestrado em Administração: uma aplicação no PPA-UEM-UEL. Revista de Gestão da USP, Vol.14, N.1, p.13-29, 2007.

WOOD, T., Jr.; CHEUKE, G.V. Ranking da produção científica em Administração de Empresas no Brasil. Revista de Administração Mackenzie, Vol.9, N.4, p.13-31, 2008 